\title{
Boundedness of $\theta$-type Calderón-Zygmund operators on Hardy spaces with non-doubling measures
}

\section{Chol $\mathrm{Ri}^{1}$ and Zhenqiu Zhang ${ }^{2^{*}}$ (D)}

\author{
"Correspondence: \\ zqzhang@nankai.edu.cn \\ ${ }^{2}$ School of Mathematical Sciences \\ and LPMC, Nankai University, \\ Tianjin, 300071, China \\ Full list of author information is \\ available at the end of the article
}

\begin{abstract}
Let $\mu$ be a non-negative Radon measure on $R^{d}$ which only satisfies some growth condition. In this paper, we obtain the boundedness of $\theta$-type Calderón-Zygmund operators on the Hardy space $H^{1}(\mu)$.
\end{abstract}

MSC: $42 \mathrm{~B} 20$

Keywords: non-doubling measure space; $\theta$-type Calderón-Zygmund operator; Hardy space

\section{Introduction and preliminaries}

During the last few decades, the theory Calderón-Zygmund operators has played a central part of modern harmonic analysis with lots of extensive applications in the other fields of mathematics. One of the most general settings to which Calderón-Zygmund theory extends naturally is the spaces of homogeneous type in the sense of Coifman and Weiss [1]. Many results from real and harmonic analysis on Euclidean spaces have their natural extensions on these spaces (see, for example, [1-3]). A metric space $(X, d)$ equipped with a non-negative Borel measure $\mu$ is called a space of homogeneous type if $(X, d, \mu)$ satisfies the measure doubling condition that there exists a positive constant $C_{\mu}$, depending on $\mu$, such that for any ball $B(x, r)=\{y \in X: d(x, y)<r\}$ with $x \in X$ and $r \in(0, \infty)$,

$$
\mu(B(x, 2 r)) \leq C_{\mu} \mu(B(x, r)) .
$$

This definition was introduced by Coifman and Weiss in [1]. The doubling condition (1.1) for measures plays a key role in the classical theory of Calderón-Zygmund operators. However, many results on the classical Calderón-Zygmund theory have been proved still valid if the doubling condition is replaced by some weaker conditions. In recent years, many papers focus on the analysis on $R^{d}$ with non-doubling measure; see [4-8] and their references. Throughout this paper, the Euclidean space $R^{d}$ is endowed with a non-negative Radon measure $\mu$ which only satisfies the following growth condition, that is, there exists $C>0$ such that

$$
\mu(B(x, r)) \leq C r^{n}
$$

(c) $2015 \mathrm{Ri}$ and Zhang. This article is distributed under the terms of the Creative Commons Attribution 4.0 International License (http://creativecommons.org/licenses/by/4.0/), which permits unrestricted use, distribution, and reproduction in any medium, provided you give appropriate credit to the original author(s) and the source, provide a link to the Creative Commons license, and indicate if changes were made. 
for all $x \in R^{d}$ and $r>0$, where $B(x, r)=\left\{y \in R^{d}:|x-y|<r\right\}, n$ is a fixed number satisfying $0<n \leq d$. Such a measure need not satisfy the doubling condition (1.1). In [6], Tolsa established Calderón-Zygmund theory for non-doubling measures.

The definition of $\theta$-type Calderón-Zygmund operator was introduced by Yabuta in [9] as follows.

Definition 1.1 Let $\theta$ be a non-negative, non-decreasing function on $R^{+}=(0, \infty)$ satisfying

$$
\int_{0}^{1} \frac{\theta(t)}{t} d t<\infty
$$

A kernel $K(\cdot, \cdot) \in L_{\text {loc }}^{1}(X \times X \backslash\{(x, y): x=y\})$ is called a $\theta$-type Calderón-Zygmund kernel if the following conditions hold:

$$
|K(x, y)| \leq C|x-y|^{-n}
$$

and

$$
\left|K(x, y)-K\left(x^{\prime}, y\right)\right|+\left|K(y, x)-K\left(y, x^{\prime}\right)\right| \leq C \theta\left(\frac{\left|x-x^{\prime}\right|}{|x-y|}\right)|x-y|^{-n}
$$

when $|x-y| \geq 2\left|x-x^{\prime}\right|$.

A linear operator $T$ is called the $\theta$-type Calderón-Zygmund operator with kernel $K(\cdot, \cdot)$ satisfying (1.4) and (1.5) if for all $f \in L^{\infty}(\mu)$ with bounded support and $x \notin \operatorname{supp} f$,

$$
T f(x)=\int_{R^{d}} K(x, y) f(y) d \mu(y) .
$$

In [10], the authors proved that the $\theta$-type Calderón-Zygmund operator which is bounded on $L^{2}(\mu)$ is also bounded from $L^{\infty}(\mu)$ into $R B M O(\mu)$ and from $H_{a t b}^{1, \infty}(\mu)$ into $L^{1}(\mu)$ on the Euclidean space with non-doubling measures.

In this paper, we discuss the boundedness of the $\theta$-type Calderón-Zygmund operator $T$ in the Hardy space $H^{1}(\mu)$. In order to state our main result, we recall some necessary notations and the known results. The following grand maximal operator was introduced by Tolsa in [11].

Definition 1.2 Given $f \in L_{\text {loc }}^{1}(\mu)$, we set

$$
M_{\Phi} f(x)=\sup _{\varphi \sim x}\left|\int_{R^{d}} f \varphi d \mu\right|
$$

where the notation $\varphi \sim x$ means that $\varphi \in L^{1}(\mu) \cap C^{1}\left(R^{d}\right)$ and satisfies

(i) $\|\varphi\|_{L^{1}(\mu)} \leq 1$,

(ii) $0 \leq \varphi(y) \leq|y-x|^{-n}$ for all $y \in R^{d}$, and

(iii) $|\nabla \varphi(y)| \leq|y-x|^{-(n+1)}$ for all $y \in R^{d}$, where $\nabla=\left(\partial / \partial x_{1}, \ldots, \partial / \partial x_{d}\right)$. 
In [11], Tolsa obtained the following result.

Theorem 1.1 A function $f$ belongs to $H_{\text {atb }}^{1, \infty}(\mu)$ if and only if $f \in L^{1}(\mu), \int f d \mu=0$ and $M_{\Phi} f \in L^{1}(\mu)$. Moreover, in this case

$$
\|f\|_{H_{a t b}^{1, \infty}(\mu)} \approx\|f\|_{L^{1}(\mu)}+\left\|M_{\Phi} f\right\|_{L^{1}(\mu)}
$$

In [12], the authors introduced a new atomic characterization of the Hardy space $H^{1}(\mu)$. Given two cubes $Q \subset R$ in $R^{d}$, set

$$
K_{Q, R}=1+\sum_{k=1}^{N_{Q, R}} \frac{\mu\left(2^{k} Q\right)}{l\left(2^{k} Q\right)^{n}},
$$

where $N_{Q, R}$ is the smallest positive integer $k$ such that $l\left(2^{k} Q\right) \geq l(R)$; see [6] for some positive of $K_{Q, R}$. The definition of the $(p, \gamma)$-atomic block is given as follows.

Definition 1.3 Let $\rho>1,1<p \leq \infty$ and $\gamma \in N$. A function $b \in L_{\text {loc }}^{1}(\mu)$ is called a $(p, \gamma)$ atomic block if

(1) there exists some cube $R$ such that $\operatorname{supp}(b) \subset R$,

(2) $\int_{R^{d}} b d(\mu)=0$,

(3) there are functions $a_{1}, a_{2}$ supported on cubes $Q_{1}, Q_{2} \subset R$ and numbers $\lambda_{1}, \lambda_{2} \in \mathbb{R}$ such that $b=\lambda_{1} a_{1}+\lambda_{2} a_{2}$, and

$$
\left\|a_{j}\right\|_{L^{p}(\mu)} \leq\left(\mu\left(\rho Q_{j}\right)\right)^{1 / p-1}\left(K_{Q_{j}, R}\right)^{-\gamma}, \quad j=1,2 .
$$

We denote $|b|_{H_{a t b, \gamma}^{1, p}(\mu)}=\left|\lambda_{1}\right|+\left|\lambda_{2}\right|$. We say that $f \in H_{a t b, \gamma}^{1, p}(\mu)$ if there are $(p, \gamma)$-atomic blocks $b_{j}$ such that

$$
f=\sum_{i=1}^{\infty} b_{i}
$$

with $\sum_{i=1}^{\infty}\left|b_{i}\right|_{H_{a t b, \gamma}^{1, p}(\mu)}<\infty$ (notice that this implies that the sum in (1.7) converges in $L^{1}(\mu)$ ). The $H_{a t b, \gamma}^{1, p}(\mu)$ norm of $f$ is defined by

$$
\|f\|_{H_{a t b, \gamma}^{1, p}(\mu)}=\inf \sum_{i=1}^{\infty}\left|b_{i}\right|_{H_{a t b, \gamma}^{1, p}(\mu)}
$$

where the infimum is taken over all the possible decompositions of $f$ into $(p, \gamma)$-atomic blocks.

We remark that the definition when $\gamma=1$ was introduced by Tolsa in [6]. It was proved in $[6,12]$ that the definition of $H_{a t b, \gamma}^{1, p}(\mu)$ is independent of the chosen constant $\rho>1$, and for any integer $\gamma \geq 1$ and $1<p \leq \infty$, all the atomic Hardy spaces $H_{a t b, \gamma}^{1, p}(\mu)$ are just the Hardy space $H_{a t}^{1, \infty}(\mu)$ with equivalent norms.

Let $T^{*}$ be the transpose of $T$. As mentioned in [13], we have to assume that $T^{*} 1=0$. Here, by $T^{*} 1=0$, we mean that for any bounded function $b$ with compact support and 


$$
\begin{aligned}
& \int_{R^{d}} b \mu=0, \\
& \int_{R^{d}} T b(x) d \mu(x)=0 .
\end{aligned}
$$

The main result of our paper is given as follows.

Theorem 1.2 Let $T$ be a $\theta$-type Calderón-Zygmund operator defined by (1.6) as above, which is bounded on $L^{2}(\mu)$ and $T^{*} 1=0$ as in (1.8). Then $T$ is bounded on $H^{1}(\mu)$.

Throughout this paper, $C$ always means a positive constant independent of the main parameters involved, but it may be different in different contents.

\section{Proof of our main result}

The following lemma will be used in the proof of Theorem 1.2.

Lemma 2.1 Let $M_{\Phi}$ be as in Definition 1.2 and $1<p<\infty$. Then $M_{\Phi}$ is bounded on $L^{p}(\mu)$.

In fact, Tolsa proved that $M_{\Phi}$ is bounded from $H^{1}(\mu)$ into $L^{1}(\mu)$; see Lemma 3.1 in [11]. On the other hand, it is obvious that $M_{\Phi}$ is bounded on $L^{\infty}(\mu)$ for $1<p<\infty$. By Theorem 7.2 in [6], we obtain that $M_{\Phi}$ is bounded on $L^{p}(\mu)$ for $1<p<\infty$.

Now we will prove Theorem 1.2.

Proof of Theorem 1.2 By the standard argument, it suffices to verify that for any atomic block $b$ as in Definition 1.3 with $\rho=4, p=\infty$ and $\gamma=2, T b$ is in $H^{1}(\mu)$ with norm $C|b|_{H_{a t b, 2}^{1, \infty}}$. By Definition 1.3, it follows

$$
\left\|a_{j}\right\|_{L^{\infty}(\mu)} \leq\left(\mu\left(4 Q_{j}\right) K_{Q_{j}, R}^{2}\right)^{-1}
$$

where $j=1,2$. The assumption that $T^{*} 1=0$ tells us that $\int_{R^{d}} \operatorname{Tb} d(\mu)=0$. Recalling that $T$ is bounded from $H^{1}(\mu)$ into $L^{1}(\mu)$ (see [6]), we obtain

$$
\|T b\|_{L^{1}(\mu)} \leq C|b|_{H_{a t b}^{1, \infty}(\mu)}
$$

By this and Theorem 1.1, we deduce that the proof of Theorem 1.2 can be reduced to proving that

$$
\left\|M_{\Phi}(T b)\right\|_{L^{1}(\mu)} \leq C|b|_{H_{a t b}^{1, \infty}(\mu)} .
$$

We can write

$$
\int_{R^{d}} M_{\Phi}(T b)(x) d \mu(x)=\int_{R^{d} \backslash 4 R} M_{\Phi}(T b)(x) d \mu(x)+\int_{4 R} M_{\Phi}(T b)(x) d \mu(x)=I_{1}+I_{2} .
$$

Let us now estimate $I_{1}$. Let $x_{R}$ be the center of the cube $R$. From the fact $T^{*} 1=0$, we obtain

$$
\begin{aligned}
I_{1} & =\int_{R^{d} \backslash 4 R} \sup _{\varphi \sim x}\left|\int_{R^{d}} T b(y)\left[\varphi(y)-\varphi\left(x_{R}\right)\right] d \mu(y)\right| d \mu(x) \\
& \leq \int_{R^{d} \backslash 4 R} \sup _{\varphi \sim x}\left|\int_{2 R} T b(y)\left[\varphi(y)-\varphi\left(x_{R}\right)\right] d \mu(y)\right| d \mu(x)
\end{aligned}
$$




$$
\begin{aligned}
& +\int_{R^{d} \backslash 4 R} \sup _{\varphi \sim x}\left|\int_{R^{d} \backslash 2 R} T b(y)\left[\varphi(y)-\varphi\left(x_{R}\right)\right] d \mu(y)\right| d \mu(x) \\
= & I_{11}+I_{12} .
\end{aligned}
$$

Note that for any $z \in 2 R, x \in 2^{k+1} R \backslash 2^{k} R$, and $k \geq 2$, we have $|x-z| \geq l\left(2^{k-2} R\right)$.

This together with Definition 1.2 and the mean value theorem leads to

$$
\left|\varphi(y)-\varphi\left(x_{R}\right)\right| \leq C \frac{l(R)}{l\left(2^{k-2} R\right)^{n+1}} .
$$

For $j=1,2$, denote $N_{Q_{j}, 2 R}$ simply by $N_{j}$ for $y \in 2 R$. By (2.3), (1.4), Hölder's inequality, the boundedness of $T$ in $L^{2}(\mu)$ and (2.1), we have

$$
\begin{aligned}
& I_{11}=\sum_{j=1}^{2}\left|\lambda_{j}\right| \sum_{k=2}^{\infty} \int_{2^{k+1} R \backslash 2^{k} R} \sup _{\varphi \sim x}\left[\int_{2 R \backslash 2 Q_{j}}\left|T a_{j}(y)\right|\left|\varphi(y)-\varphi\left(x_{R}\right)\right| d \mu(y)\right] d \mu(x) \\
& +\sum_{j=1}^{2}\left|\lambda_{j}\right| \sum_{k=2}^{\infty} \int_{2^{k+1} R \backslash 2^{k} R} \sup _{\varphi \sim x}\left[\int_{2 Q_{j}}\left|T a_{j}(y)\right|\left|\varphi(y)-\varphi\left(x_{R}\right)\right| d \mu(y)\right] d \mu(x) \\
& \leq C \sum_{j=1}^{2}\left|\lambda_{j}\right| \sum_{k=2}^{\infty} \int_{2^{k+1} R \backslash 2^{k} R} \frac{l(R)}{l\left(2^{k-2} R\right)^{n+1}} \sum_{l=1}^{N_{j}-1} \int_{2^{l+1} Q_{j} \backslash 2^{l} Q_{j}} \int_{Q_{j}} \frac{\left|a_{j}(z)\right|}{|y-z|^{n}} d \mu(z) d \mu(y) d \mu(x) \\
& +C \sum_{j=1}^{2}\left|\lambda_{j}\right| \sum_{k=2}^{\infty} \int_{2^{k+1} R \backslash 2^{k} R} \frac{l(R)}{l\left(2^{k-2} R\right)^{n+1}}\left\|\left(T a_{j}\right) \chi_{2 Q_{j}}\right\|_{L^{1}(\mu)} d \mu(x) \\
& \leq C \sum_{j=1}^{2}\left|\lambda_{j}\right| \sum_{k=2}^{\infty} 2^{-k} \sum_{l=1}^{N_{j}-1} \frac{\mu\left(2^{l+1} Q_{j}\right)}{l\left(2^{l+1} Q_{j}\right)^{n}}\left\|a_{j}\right\|_{L^{\infty}(\mu)} \mu\left(Q_{j}\right) \\
& +C \sum_{j=1}^{2}\left|\lambda_{j}\right| \sum_{k=2}^{\infty} 2^{-k}\left\|\left(T a_{j}\right) \chi_{2 Q_{j}}\right\|_{L^{2}(\mu)} \mu\left(2 Q_{j}\right)^{1 / 2} \\
& \leq C \sum_{j=1}^{2}\left|\lambda_{j}\right| K_{Q_{j}, R}\left\|a_{j}\right\|_{L^{\infty}(\mu)} \mu\left(Q_{j}\right)+C \sum_{j=1}^{2}\left|\lambda_{j}\right|\left\|a_{j}\right\|_{L^{2}(\mu)} \mu\left(2 Q_{j}\right)^{1 / 2} \\
& \leq C \sum_{j=1}^{2}\left|\lambda_{j}\right|
\end{aligned}
$$

where we have used the fact that

$$
K_{Q_{j}, 2 R} \leq C K_{Q_{j}, R}
$$

For $I_{12}$, we get

$$
\begin{aligned}
I_{12} & =\sum_{k=2}^{\infty} \int_{2^{k+1} R \backslash 2^{k} R \varphi \sim x} \sup _{R^{d} \backslash 2 R} T b(y)\left[\varphi(y)-\varphi\left(x_{R}\right)\right] d \mu(y) \mid d \mu(x) \\
& \leq \sum_{k=2}^{\infty} \int_{2^{k+1} R \backslash 2^{k} R} M_{\Phi}\left[|T b| \chi_{2^{k+2} R \backslash 2^{k-1} R}\right](x) d \mu(x)
\end{aligned}
$$




$$
\begin{aligned}
& +\sum_{k=2}^{\infty} \int_{2^{k+1} R \backslash 2^{k} R} \sup _{\varphi \sim x}\left[\int_{2^{k+2} R \backslash 2^{k-1} R}|T b(y)| \varphi\left(x_{R}\right) d \mu(y)\right] d \mu(x) \\
& +\sum_{k=2}^{\infty} \int_{2^{k+1} R \backslash 2^{k} R} \sup _{\varphi \sim x}\left[\int_{R^{d} \backslash 2^{k+2} R}|T b(y)|\left(\varphi(y)+\varphi\left(x_{R}\right)\right) d \mu(y)\right] d \mu(x) \\
& +\sum_{k=2}^{\infty} \int_{2^{k+1} R \backslash 2^{k} R \varphi \sim x} \sup _{\varphi \sim}\left[\int_{2^{k-1} R \backslash 2 R}|T b(y)|\left(\varphi(y)+\varphi\left(x_{R}\right)\right) d \mu(y)\right] d \mu(x) \\
& =I_{121}+I_{122}+I_{123}+I_{124} .
\end{aligned}
$$

From Lemma 2.1, the fact that $\int_{R^{d}} b d(\mu)=0$ and (1.5), we can deduce that

$$
\begin{aligned}
I_{121} \leq & \sum_{k=2}^{\infty} \mu\left(2^{k+1} R\right)^{1 / 2}\left\|M_{\Phi}\left[|T b| \chi_{2^{k+2} R \backslash 2^{k-1} R}\right]\right\|_{L^{2}(\mu)} \\
\leq & C \sum_{k=2}^{\infty} \mu\left(2^{k+1} R\right)^{1 / 2}\left(\int_{2^{k+2} R \backslash 2^{k-1} R}\left|\int_{R}\left(K(y, z)-K\left(y, x_{R}\right)\right) b(z) d \mu(z)\right|^{2} d \mu(y)\right)^{1 / 2} \\
\leq & C \sum_{k=2}^{\infty} \mu\left(2^{k+1} R\right)^{1 / 2} \\
& \times\left(\int_{2^{k+2} R \backslash 2^{k-1} R}\left[\int_{R} \theta\left(\frac{\left|z-x_{R}\right|}{\left|y-x_{R}\right|}\right)\left|y-x_{R}\right|^{-n}|b(z)| d \mu(z)\right]^{2} d \mu(y)\right)^{1 / 2} \\
\leq & C \sum_{k=2}^{\infty} \frac{\mu\left(2^{k+1} R\right)}{l\left(2^{k} R\right)^{n}} \theta\left(2^{-k}\right)\|b\|_{L^{1}(\mu)} \leq C \int_{0}^{1} \frac{\theta(t)}{t} d t\|b\|_{L^{1}(\mu)} \leq C \sum_{j=1}^{2}\left|\lambda_{j}\right|,
\end{aligned}
$$

where we have used the following inequality:

$$
\int_{0}^{1} \frac{\theta(t)}{t} \geq \sum \int_{2^{k}}^{2^{1-k}} \frac{\theta\left(2^{-k}\right)}{2^{1-k}} \geq C \sum_{k=1}^{\infty} \theta\left(2^{-k}\right),
$$

and the fact

$$
\|b\|_{L^{1}(\mu)} \leq \sum_{j=1}^{2}\left|\lambda_{j}\right|\left\|a_{j}\right\|_{L^{1}(\mu)} \leq C \sum_{j=1}^{2}\left|\lambda_{j}\right| .
$$

An argument similar to the estimate for $I_{121}$ tells us that

$$
I_{122} \leq C \sum_{j=1}^{2}\left|\lambda_{j}\right|
$$

Finally, we estimate $I_{123}$. By the fact that $\int_{R^{d}} b d \mu=0$, Definition 1.2 and (1.5), we obtain

$$
\begin{aligned}
I_{123} \leq & \sum_{k=2}^{\infty} \int_{2^{k+1} R \backslash 2^{k} R_{l=k+2}} \sum_{2^{l+1} R \backslash 2^{l} R} \int_{R}\left|K(y, z)-K\left(y, x_{R}\right)\right||b(z)| d \mu(z) \\
& \times\left[\frac{1}{|y-x|^{n}}+\frac{1}{\left|x_{R}-x\right|^{n}}\right] d \mu(y) d \mu(x)
\end{aligned}
$$




$$
\begin{aligned}
\leq & C \sum_{k=2}^{\infty} \int_{2^{k+1} R \backslash 2^{k} R} \sum_{l=k+2}^{\infty} \int_{2^{l+1} R \backslash 2_{R}{ }_{R}} \int_{R} \theta\left(\frac{\left|z-x_{R}\right|}{\left|y-x_{R}\right|}\right)\left|y-x_{R}\right|^{-n}|b(z)| d \mu(z) \\
& \times\left[\frac{1}{|y-x|^{n}}+\frac{1}{\left|x_{R}-x\right|^{n}}\right] d \mu(y) d \mu(x) \\
\leq & C \sum_{k=2}^{\infty} \sum_{l=k+2}^{\infty} \theta\left(2^{-l}\right) \frac{\mu\left(2^{l+1} R\right)}{l\left(2^{l+1} R\right)^{n}} \frac{\mu\left(2^{k+1} R\right)}{l\left(2^{k+1} R\right)^{n}}\|b\|_{L^{1}(\mu)} \\
\leq & C \sum_{j=1}^{2}\left|\lambda_{j}\right| .
\end{aligned}
$$

An argument similar to the estimate for $I_{123}$ indicates that

$$
I_{124} \leq C \sum_{j=1}^{2}\left|\lambda_{j}\right| .
$$

Combining the estimate for $I_{121}, I_{122}, I_{123}$ and $I_{124}$, we obtain the desired estimate for $I_{12}$. The estimates for $I_{11}$ and $I_{12}$ tell us that

$$
I_{1}=\int_{R^{d} \backslash 4 R} M_{\Phi}(T b)(x) d \mu(x) \leq C|b|_{H_{a t b, 2}^{1, \infty}}(\mu) .
$$

For $I_{2}$, by the sublinearity of $M_{\Phi}$, it follows

$$
I_{2} \leq \int_{4 R} M_{\Phi}\left[(T b) \chi_{8 R}\right](x) d \mu(x)+\int_{4 R} M_{\Phi}\left[(T b) \chi_{R^{d} \backslash 8 R}\right](x) d \mu(x)=I_{21}+I_{22} .
$$

From $Q_{j} \subset R$, Definition 1.2 and (2.1), we obtain

$$
\begin{aligned}
I_{22} & \leq \int_{4 R} \sup _{\varphi \sim x}\left[\int_{R^{d} \backslash 8 R}|T b(y)| \varphi(y) d \mu(y)\right] d \mu(x) \\
& \leq \sum_{j=1}^{2}\left|\lambda_{j}\right| \int_{4 R} \sum_{k=2}^{\infty} \int_{2^{k+1} R \backslash 2^{k} R}\left|\int_{Q_{j}} K(y, z) a_{j}(z) d \mu(z)\right| \frac{1}{|x-y|^{n}} d \mu(y) d \mu(x) \\
& \leq C \sum_{j=1}^{2}\left|\lambda_{j}\right| \sum_{k=3}^{\infty}\left\|a_{j}\right\|_{L^{\infty}(\mu)} \mu\left(Q_{j}\right) \frac{\mu\left(2^{k+1} R\right)}{l\left(2^{k-2} R\right)^{n}} \frac{\mu(4 R)}{l\left(2^{k-2} R\right)^{n}} \\
& \leq C \sum_{j=1}^{2}\left|\lambda_{j}\right| .
\end{aligned}
$$

In order to estimate $I_{21}$, we write

$$
\begin{aligned}
I_{21} \leq & \sum_{j=1}^{2}\left|\lambda_{j}\right| \int_{4 Q_{j}} M_{\Phi}\left[\left(T a_{j}\right) \chi_{8 R}\right](x) d \mu(x)+\sum_{j=1}^{2}\left|\lambda_{j}\right| \int_{4 R \backslash 4 Q_{j}} M_{\Phi}\left[\left(T a_{j}\right) \chi_{2 Q_{j}}\right](x) d \mu(x) \\
& +\sum_{j=1}^{2}\left|\lambda_{j}\right| \int_{4 R \backslash 4 Q_{j}} M_{\Phi}\left[\left(T a_{j}\right) \chi_{8 R \backslash 2 Q_{j}}\right](x) d \mu(x) \\
& =I_{211}+I_{212}+I_{213} .
\end{aligned}
$$


Hölder's inequality, Lemma 2.1, the boundedness of $T$ in $L^{2}(\mu)$ and (2.1) lead to

$$
\begin{aligned}
I_{211} & \leq \sum_{j=1}^{2}\left|\lambda_{j}\right| \mu\left(4 Q_{j}\right)^{1 / 2}\left\|M_{\Phi}\left[\left(T a_{j}\right) \chi_{8 R}\right]\right\|_{L^{2}(\mu)} \\
& \leq C \sum_{j=1}^{2}\left|\lambda_{j}\right| \mu\left(4 Q_{j}\right)^{1 / 2}\left\|T a_{j}\right\|_{L^{2}(\mu)} \leq C \sum_{j=1}^{2}\left|\lambda_{j}\right| \mu\left(4 Q_{j}\right)^{1 / 2}\left\|a_{j}\right\|_{L^{2}(\mu)} \\
& \leq C \sum_{j=1}^{2}\left|\lambda_{j}\right| \mu\left(4 Q_{j}\right)\left\|a_{j}\right\|_{L^{\infty}(\mu)} \leq C \sum_{j=1}^{2}\left|\lambda_{j}\right| .
\end{aligned}
$$

By Definition 1.2, Hölder's inequality, the boundedness of $T$ in $L^{2}(\mu)$ and (2.1), we get

$$
\begin{aligned}
I_{212} & \leq \sum_{j=1}^{2}\left|\lambda_{j}\right| \sum_{k=2}^{N_{Q_{j}, 4 R}} \int_{2^{k+1} Q_{j} \backslash 2^{k} Q_{j}} \sup _{\varphi \sim x}\left|\int_{2 Q_{j}} \operatorname{Ta}_{j}(y) \varphi(y) d \mu(y)\right| d \mu(x) \\
& \leq \sum_{j=1}^{2}\left|\lambda_{j}\right| \sum_{k=2}^{N_{Q_{j}, 4 R}} \int_{2^{k+1} Q_{j} \backslash 2^{k} Q_{j}} \frac{1}{l\left(2^{k-2} Q_{j}\right)^{n}} d \mu(x) \int_{2 Q_{j}}\left|T a_{j}(y)\right| d \mu(y) \\
& \leq \sum_{j=1}^{2}\left|\lambda_{j}\right| \sum_{k=2}^{N_{Q_{j}, 4 R}} \frac{\mu\left(2^{k+1} Q_{j}\right)}{l\left(2^{k-2} Q_{j}\right)^{n}}\left\|T a_{j}\right\|_{L^{2}(\mu)} \mu\left(2 Q_{j}\right)^{1 / 2} \\
& \leq C \sum_{j=1}^{2}\left|\lambda_{j}\right| K_{Q_{j}, R} \mu\left(2 Q_{j}\right)^{1 / 2}\left\|a_{j}\right\|_{L^{2}(\mu)} \\
& \leq C \sum_{j=1}^{2}\left|\lambda_{j}\right|,
\end{aligned}
$$

where we have used the fact that

$$
K_{Q_{j}, 4 R} \leq C K_{Q_{j}, R}
$$

For $I_{213}$, we can write

$$
\begin{aligned}
I_{213}= & \sum_{j=1}^{2}\left|\lambda_{j}\right| \sum_{k=2}^{N_{Q_{j}, 4 R}} \int_{2^{k+1} Q_{j} \backslash 2^{k} Q_{j}} M_{\Phi}\left[\left(T a_{j}\right) \chi_{8 R \backslash 2 Q_{j}}\right](x) d \mu(x) \\
\leq & \sum_{j=1}^{2}\left|\lambda_{j}\right| \sum_{k=2}^{N_{Q_{j}, 4 R}} \int_{2^{k+1} Q_{j} \backslash 2^{k} Q_{j}} M_{\Phi}\left[\left|T a_{j}\right| \chi_{2^{k+2} Q_{j} \backslash 2^{k-1} Q_{j}}\right](x) d \mu(x) \\
& +\sum_{j=1}^{2}\left|\lambda_{j}\right| \sum_{k=2}^{N_{Q_{j}, 4 R}} \int_{2^{k+1} Q_{j} \backslash 2^{k} Q_{j}} M_{\Phi}\left[\left|T a_{j}\right| \chi_{\max \left\{2^{k+2} Q_{j}, 8 R\right\} \backslash 2^{k+2} Q_{j}}\right](x) d \mu(x) \\
& +\sum_{j=1}^{2}\left|\lambda_{j}\right| \sum_{k=2}^{N_{Q_{j}, 4 R}} \int_{2^{k+1} Q_{j} \backslash 2^{k} Q_{j}} M_{\Phi}\left[\left|T a_{j}\right| \chi_{2^{k-1} Q_{j} \backslash 2 Q_{j}}\right](x) d \mu(x) \\
= & J_{1}+J_{2}+J_{3} .
\end{aligned}
$$


Lemma 2.1, (1.4) and (2.1) imply that

$$
\begin{aligned}
J_{1} & =\sum_{j=1}^{2}\left|\lambda_{j}\right| \sum_{k=2}^{N_{Q_{j}, 4 R}} \mu\left(2^{k+1} Q_{j}\right)^{1 / 2}\left\|M_{\Phi}\left[f\left|T a_{j}\right| \chi_{2^{k+2} Q_{j} \backslash 2^{k-1} Q_{j}}\right]\right\|_{L^{2}(\mu)} \\
& \leq C \sum_{j=1}^{2}\left|\lambda_{j}\right| \sum_{k=2}^{N_{Q_{j}, 4 R}} \mu\left(2^{k+1} Q_{j}\right)^{1 / 2} \times\left(\int_{2^{k+2} Q_{j} \backslash 2^{k-1} Q_{j}}\left|\int_{Q_{j}} K(y, z) a_{j}(z) d \mu(z)\right|^{2} d \mu(y)\right)^{1 / 2} \\
& \leq C \sum_{j=1}^{2}\left|\lambda_{j}\right| \sum_{k=2}^{N_{Q_{j}, 4 R}} \frac{\mu\left(2^{k+2} Q_{j}\right)}{l\left(2^{k-3} Q_{j}\right)^{n}}\left\|a_{j}\right\|_{L^{\infty}(\mu)} \mu\left(Q_{j}\right) \\
& \leq C \sum_{j=1}^{2}\left|\lambda_{j}\right| .
\end{aligned}
$$

By (ii) of Definition 1.2, (1.4), (2.5) and (2.1), we have

$$
\begin{aligned}
J_{2} & =\sum_{j=1}^{2}\left|\lambda_{j}\right| \sum_{k=2}^{N_{Q_{j}, 4 R}} \int_{2^{k+1} Q_{j} \backslash 2^{k} Q_{j}} \sup _{\varphi \sim x}\left[\int_{2^{k-1} Q_{j} \backslash 2 Q_{j}}\left|T a_{j}(y)\right| \varphi(y) d \mu(y)\right] d \mu(x) \\
& \leq \sum_{j=1}^{2}\left|\lambda_{j}\right| \sum_{k=2}^{N_{Q_{j}, 4 R}} \int_{2^{k+1} Q_{j} \backslash 2^{k} Q_{j}} \sum_{l=1}^{k-2} \int_{2^{l+1} Q_{j} \backslash 2^{l} Q_{j}}\left|\int_{Q_{j}} K(y, z) a_{j}(z) d \mu(z)\right| \frac{1}{|y-x|^{n}} d \mu(y) d \mu(x) \\
& \leq C \sum_{j=1}^{2}\left|\lambda_{j}\right| \sum_{k=2}^{N_{Q_{j}, 4 R}} \frac{\mu\left(2^{k+1} Q_{)}\right)}{l\left(2^{k+1} Q_{j}\right)^{n}} \sum_{l=1}^{k-2} \frac{\mu\left(2^{l+1} Q_{)}\right.}{l\left(2^{l+1} Q_{j}\right)^{n}}\left\|a_{j}\right\|_{L^{\infty}(\mu)} \mu\left(Q_{j}\right) \\
& \leq C \sum_{j=1}^{2}\left|\lambda_{j}\right|\left(K_{Q_{j}, R}\right)^{2}\left\|a_{j}\right\|_{L^{\infty}(\mu)} \mu\left(Q_{j}\right) \\
& \leq C \sum_{j=1}^{2}\left|\lambda_{j}\right| .
\end{aligned}
$$

With the argument similar to the estimate for $J_{2}$ it follows that

$$
J_{3}=\sum_{j=1}^{2}\left|\lambda_{j}\right| \sum_{k=2}^{N_{Q_{j}, 4 R}} \int_{2^{k+1} Q_{j} \backslash 2^{k} Q_{j}} M_{\Phi}\left[\left|T a_{j}\right| \chi_{2^{k-1} Q_{j} \backslash 2 Q_{j}}\right](x) d \mu(x) \leq C \sum_{j=1}^{2}\left|\lambda_{j}\right| .
$$

Thus

$$
I_{213}=\sum_{j=1}^{2}\left|\lambda_{j}\right| \sum_{k=2}^{N_{Q_{j}, 4 R}} \int_{2^{k+1} Q_{j} \backslash 2^{k} Q_{j}} M_{\Phi}\left[\left(T a_{j}\right) \chi_{8 R \backslash 2 Q_{j}}\right](x) d \mu(x) \leq C \sum_{j=1}^{2}\left|\lambda_{j}\right| .
$$

From the estimation of $I_{21}$ and $I_{22}$, we obtain

$$
I_{2}=\int_{4 R} M_{\Phi}(T b)(x) d \mu(x) \leq C \sum_{j=1}^{2}\left|\lambda_{j}\right|=C|b|_{H_{a t b, 2}^{1, \infty}}
$$


The estimates (2.4) and (2.6) lead to (2.2), and this completes the proof of our theorem.

Remark 2.2 It is known that the dual space of $H^{1}(\mu)$ is the space $R B M O(\mu)$, which is introduced in [12]. From Theorem 1.2, the fact that $R B M O(\mu)=\left(H^{1}(\mu)\right)^{*}$ and a standard dual argument, it is easy to deduce the boundedness of the transpose operator of $T$ on the $R B M O(\mu)$ space as below.

Corollary 2.3 Let $T$ be the same as in Theorem 1.2. Then $T^{*}$, the transpose operator of $T$, is bounded on $R B M O(\mu)$.

\section{Competing interests}

The authors declare that they have no competing interests.

\section{Authors' contributions}

All authors contributed equally to the writing of this paper. All authors read and approved the final manuscript.

\section{Author details}

${ }^{1}$ Department of Mathematics, Kim Hyong Jik Normal University, Pyong Yang, D.P.R of Korea. ${ }^{2}$ School of Mathematical Sciences and LPMC, Nankai University, Tianjin, 300071, China.

\section{Acknowledgements}

This work was supported in part by the National Natural Science Foundation of China (Grant No. 11271091).

Received: 28 April 2015 Accepted: 28 September 2015 Published online: 09 October 2015

\section{References}

1. Coifman, R, Weiss, G: Analyse harmonique non-commutative sur certains espaces homogènes. Lecture Notes in Mathematics, vol. 242. Springer, Berlin (1971)

2. Coifman, R, Weiss, G: Extensions of Hardy spaces and their use in analysis. Bull. Am. Math. Soc. 83, $569-645$ (1977)

3. Heinenon, J: Lectures on Analysis on Metric Spaces. Springer, Berlin (2001)

4. Fu, X, Hu, G, Yang, D: A remark on the boundedness of Calderón-Zygmund operators in non-homogeneous spaces. Acta Math. Sin. Engl. Ser. 23, 449-456 (2007)

5. Nazarov, F, Treil, S, Volberg, A: The Tb-theorem on non-homogeneous spaces. Acta Math. 190, 151-239 (2003)

6. Tolsa, X: BMO, $H^{1}$ and Calderón-Zygmund operators for non doubling measures. Math. Ann. 319, 89-149 (2001)

7. Tolsa, X: Littlewood-Paley theory and the T(1) theorem with non-doubling measures. Adv. Math. 164, 57-116 (2001)

8. Yang, D, Yang, D, Hu, G: The Hardy Space $H^{1}$ with Non-doubling Measures and Their Applications. Lecture Notes in Mathematics, vol. 2084. Springer, Berlin (2013)

9. Yabuta, K: Generalization of Calderón-Zygmund operators. Stud. Math. 82, 17-31 (1985)

10. Xie, R, Shu, L: $\theta$-Type Calderón-Zygmund operators with non-doubling measures. Acta Math. Appl. Sinica (Engl. Ser.) 29, 263-280 (2013)

11. Tolsa, $X$ : The space $H^{1}$ for nondoubling measures in terms of a grand maximal operator. Trans. Am. Math. Soc. 355 , 315-348 (2003)

12. Hu, G, Meng, Y, Yang, D: New atomic characterization of $H^{1}$ space with non-doubling measures and its applications. Math. Proc. Camb. Philos. Soc. 138, 151-171 (2005)

13. Chen, W, Meng, Y, Yang, D: Calderón-Zygmund operators on Hardy space without the doubling condition. Proc. Am. Math. Soc. 133, 2671-2680 (2005)

\section{Submit your manuscript to a SpringerOpen ${ }^{\odot}$ journal and benefit from:}

- Convenient online submission

Rigorous peer review

- Immediate publication on acceptance

- Open access: articles freely available online

- High visibility within the field

- Retaining the copyright to your article 